\title{
SEMISTABILITY OF LAZARSFELD-MUKAI BUNDLES VIA PARABOLIC STRUCTURES
}

\author{
POORNAPUSHKALA NARAYANAN
}

\begin{abstract}
Our aim in this article is to produce new examples of semistable LazarsfeldMukai bundles on smooth projective surfaces $X$ using the notion of parabolic vector bundles. In particular, we associate natural parabolic structures to any rank two (dual) Lazarsfeld-Mukai bundle and study the parabolic stability of these parabolic bundles. We also show that the orbifold bundles on Kawamata coverings of $X$ corresponding to the above parabolic bundles are themselves certain (dual) Lazarsfeld-Mukai bundles. This gives semistable Lazarsfeld-Mukai bundles on Kawamata covers of the projective plane and of certain K3 surfaces.
\end{abstract}

\section{InTRODUCTION}

In recent years, the study of certain vector bundles called Lazarsfeld-Mukai bundles (LM bundles) has gained prominence. Investigating the properties of these naturally occurring bundles is of increasing interest. This can be attributed to the deep applications of these bundles in Brill-Noether theory of curves, especially those lying on K3 surfaces.

LM bundles were first used by Lazarsfeld [11] to prove Petri's conjecture and by Mukai [16] in the classification of certain Fano manifolds. They have also been useful in studying the constancy of gonality, Clifford index and Clifford dimension of smooth projective curves belonging to ample or globally generated linear systems on K3 surfaces [5, 6, 9]. Voisin's proof of the generic Green's conjecture employed these bundles [21, 22], and Aprodu and Farkas [1] use LM bundles and their parameter spaces while proving Green's conjecture for curves on a K3 surface. The (semi)stability properties of LM bundles over K3 surfaces were studied by Lelli-Chiesa [13], and of certain LM bundles over Jacobian surfaces and higher dimensional varieties by us [18, 19].

In this article, our aim is to produce new examples of semistable Lazarsfeld-Mukai bundles via the general theory of parabolic vector bundles. In particular, we associate certain parabolic vector bundles to a rank two LM bundle and study the related notions of parabolic stability. We refer to $\S 2$ for some preliminaries on parabolic vector bundles.

Suppose $X$ is a smooth projective surface over $\mathbb{C}$ and $C \stackrel{i}{\hookrightarrow} X$ is a smooth curve. Consider a globally generated line bundle $A$ on $C$, with $\operatorname{dim} H^{0}(C, A)=2$. Then the rank two LM bundle associated to the pair $(C, A)$ is the dual of the vector bundle $F$, where $F$ is defined by the following short exact sequence:

$$
0 \rightarrow F \rightarrow H^{0}(C, A) \otimes \mathcal{O}_{X} \rightarrow i_{*} A \rightarrow 0 .
$$

Key words and phrases. (Semi)stability, Parabolic bundles.

Mathematics Classification numbers: 14C20, 14E20, 14J60. 
Note that $F$ comes in-built with a parabolic structure given by the following filtration with associated weights $0 \leq a_{1}<a_{2}<1$ :

$$
\mathfrak{F}_{C}:\left.\left.F\right|_{C} \supset_{a_{1}} A \otimes \mathcal{O}_{X}(-C)\right|_{C} \supset_{a_{2}} 0 .
$$

We thus obtain a parabolic vector bundle $F_{*}=\left(F, \mathfrak{F}_{C}, a_{1}, a_{2}\right)$ which we call the (dual) parabolic LM bundle.

Our second parabolic bundle arises as follows. Consider a point $q$ of multiplicity one on $C$. Consider the blow up of $X$ at $q$, say $\widetilde{X}$. Let $\pi: \widetilde{X} \rightarrow X$ denote the blow down map and $E \subset \widetilde{X}$, the exceptional divisor. The pulled back rank two vector bundle $\widetilde{F}:=\pi^{*} F$ on $\widetilde{X}$ admits a parabolic structure along the exceptional divisor $E$ by associating weights $0 \leq b_{1}<b_{2}<1$ :

$$
\widetilde{\mathfrak{F}}_{E}:\left.\widetilde{F}\right|_{E}=F(q) \otimes \mathcal{O}_{E} \supset_{b_{1}} M \supset_{b_{2}} 0 .
$$

Here, $M=\operatorname{kernel}\left(\left.\widetilde{F}\right|_{E} \rightarrow H^{0}(C, A) \otimes \mathcal{O}_{E}\right)$. We call $\widetilde{F}_{*}:=\left(\widetilde{F}, \widetilde{F}_{E}, b_{1}, b_{2}\right)$ the (dual) blown up parabolic LM bundle. Refer $\S 3$ for details.

In $\S$, we study the parabolic stability of the two parabolic vector bundles mentioned above. Let $L$ be an ample line bundle on $X$. Then we have the following theorem.

Theorem 1.1. Suppose that the vector bundle $F$ is $\mu_{L}$-stable. Then the (dual) parabolic $L M$ bundle $F_{*}$ on $X$ is parabolic $\mu_{L}$-stable if the weights $a_{1}, a_{2}$ are such that:

(a) $a_{2}-a_{1}<\frac{2}{(C \cdot L)}$, if the intersection number $(C \cdot L)$ is even, or

(b) $a_{2}-a_{1}<\frac{1}{(C \cdot L)}$, if $(C \cdot L)$ is odd.

Next, consider the line bundle $L_{n}:=n \pi^{*} L \otimes \mathcal{O}_{\widetilde{X}}(-E)$ on $\widetilde{X}$ which is ample for $n$ sufficiently large. In this case, we have the following theorem.

Theorem 1.2. Suppose that $F$ is $\mu_{L}$-stable. Then, there is an integer $n_{0}$ such that for all $n \geq n_{0}, \widetilde{F}_{*}$ is parabolic $\mu_{L_{n}}$-stable for all weights $b_{1}$ and $b_{2}$.

In [3], Biswas established a two way relationship between parabolic vector bundles and orbifold vector bundles. Motivated by this, in $\S 5$, we obtain the orbifold bundle on Kawamata covers of $X$ associated to the (dual) parabolic LM bundle $F_{*}$ for weights of the form $a_{1}=0$ and $a_{2}=\frac{N-m}{N}$, where $1 \leq m<N$ are positive integers. Let $p: Y \rightarrow X$ be the Kawamata covering of $X$ such that $p^{*} C$ is a non-reduced divisor $N C^{\prime}$ with $C^{\prime}:=\left(p^{*} C\right)_{\text {red }}$ a smooth curve on $Y$. Then we have the following theorem.

Theorem 1.3. The orbifold vector bundle on $Y$ corresponding to the (dual) parabolic LM bundle $\left(F, \mathfrak{F}_{C}, 0, \frac{N-m}{N}\right)$ on $X$ is the dual LM bundle on $Y$, say $F^{\prime}$, associated to the triple $\left(m C^{\prime}, A^{\prime}, H^{0}(C, A)\right)$ given by the short exact sequence

$$
0 \rightarrow F^{\prime} \rightarrow H^{0}(C, A) \otimes \mathcal{O}_{Y} \rightarrow j_{*}^{\prime} A^{\prime} \rightarrow 0 .
$$

Here $A^{\prime}$ is the pullback of the line bundle $A$ from $C$ to the curve $m C^{\prime} \stackrel{j^{\prime}}{\rightarrow} Y$ (the curve $m C^{\prime}$ is non-reduced when $m>1$ ).

It is interesting to observe that the orbifold bundles are themselves certain dual LM bundles on the Kawamata covers. This further highlights that LM bundles are vector bundles with desirable properties. 
As a consequence of the above correspondence, we obtain new examples of semistable LM bundles on the Kawamata covers of $\mathbb{P}^{2}$ and K3 surfaces in $\S 6$.

Theorem 1.4. Suppose $X$ is a smooth projective surface and $C$ is a smooth curve on $X$. Let $A$ be an ample line bundle on $C$ and $V \subset H^{0}(C, A)$ be a general two dimensional subspace with the linear series $\mathbb{P} V$ base-point free. For any positive integer $N$, consider the Kawamata covering $p: Y \rightarrow X$ of $X$ such that $p^{*} C$ is a non-reduced divisor $N C^{\prime}$ with $C^{\prime}:=\left(p^{*} C\right)_{\text {red }}$ a smooth curve on $Y$. Let $1 \leq m<N$ be an integer and $A^{\prime}$ denote the pullback of $A$ from $C$ to (the possibly non-reduced curve) $m C^{\prime}$.

(a) Consider $X=\mathbb{P}^{2}$ and $C \in|\mathcal{O}(d)|$, where $d$ is odd. Set $A$ to be of the form $\left.\mathcal{O}(r d)\right|_{C}$ for $r \geq 1$. Choose $m$ such that $\frac{N-m}{N}<\frac{1}{d}$. Then the LM bundle on $Y$ associated to the triple $\left(m C^{\prime}, A^{\prime}, V\right)$ is $\mu_{p^{*} \mathcal{O}(1)}$-semistable.

(b) Let $X$ be a smooth projective K3 surface and $L$ be an ample line bundle on $X$ such that a general curve $C \in|L|$ has genus $g$, Clifford dimension 1 and maximal gonality. Let $A$ be a complete base-point free $g_{d}^{1}$ on $C$ where the Brill Noether number $\rho(g, 1, d)>0$. Set $V=H^{0}(C, A)$. Let $m$ be such that $\frac{N-m}{N}<\frac{1}{g-1}$. Then the LM bundle on $Y$ associated to the triple $\left(m C^{\prime}, A^{\prime}, H^{0}(C, A)\right)$ is $\mu_{p^{*} L^{-}}$semistable.

We have the following as particular cases of (a) and (b) respectively. Let $l=\operatorname{deg} A^{\prime}$.

(a') Let $d=1$, i.e. $C \in|\mathcal{O}(1)|$ is a line and set $m=1$. Then there is an irreducible component of the Brill-Noether variety $\mathcal{G}_{l}^{1}\left(\left|\mathcal{O}_{Y}\left(C^{\prime}\right)\right|\right)$ corresponding to $\mu_{p^{*} \mathcal{O}(1)}$-semistable LM bundles.

(b') Suppose $X$ be a smooth projective $K 3$ surface and $L$ is as above which in addition satisfies $\left(L^{2}\right)=2$. Let $m=1$. Then there is an irreducible component of the Brill-Noether variety $\mathcal{G}_{l}^{1}\left(\left|\mathcal{O}_{Y}\left(C^{\prime}\right)\right|\right)$ corresponding to $\mu_{p^{*} L^{-}}$semistable LM bundles.

Acknowledgements. I thank Dr. Jaya NN Iyer for introducing me to this problem and for guiding me during the course of this project. I also thank Dr. T. E. Venkata Balaji and Prof. D. S. Nagaraj for helpful discussions.

\section{Preliminaries}

2.1. Parabolic sheaves. Parabolic bundles were first introduced over curves by Seshadri [20] and Mehta [15]. This was generalized to higher dimensions by Bhosle [2] and by Maruyama-Yokogawa [14]. Consider a connected smooth projective variety $X$ and an effective divisor $D$ on $X$.

Definition 2.1. Let $E$ be a coherent torsion-free $\mathcal{O}_{X}$-module. A quasi-parabolic structure on $E$ along $D$ is defined by giving a filtration of $\left.E\right|_{D}$ of the form:

$$
\mathfrak{F}_{D}:\left.E\right|_{D}=F_{1}\left(\left.E\right|_{D}\right) \supset F_{2}\left(\left.E\right|_{D}\right) \supset \cdots \supset F_{l}\left(\left.E\right|_{D}\right) \supset F_{l+1}\left(\left.E\right|_{D}\right)=0 .
$$

A parabolic structure on $E$ is a quasi-parabolic structure as above together with a system of parabolic weights $a_{1}, a_{2}, \cdots, a_{l}$ such that $0 \leq a_{1}<a_{2}<\cdots<a_{l}<1$.

We denote $E$ with the parabolic structure by $\left(E, \mathfrak{F}_{D}, a_{*}\right)$ or by $E_{*}$. If $E$ is locally free, then $E_{*}$ is called a parabolic bundle. 
In the following definitions, let $E$ be a torsion-free coherent sheaf with parabolic structure $\left(E, \mathfrak{F}_{D}, a_{i}(E)\right)$ where $i=1,2, \ldots, l$ and

$$
\mathfrak{F}_{D}:\left.E\right|_{D}=F_{1}\left(\left.E\right|_{D}\right) \supset F_{2}\left(\left.E\right|_{D}\right) \supset \cdots \supset F_{l}\left(\left.E\right|_{D}\right) \supset F_{l+1}\left(\left.E\right|_{D}\right)=0 .
$$

Definition 2.2. [2, Definition 1.10] Suppose that $G$ is another torsion-free coherent sheaf with quasi-parabolic structure along $D$ prescribed by the filtration $\mathfrak{F}_{D}^{\prime}$ of $\left.G\right|_{D}$ :

$$
\mathfrak{F}_{D}^{\prime}:\left.G\right|_{D}=F_{1}\left(\left.G\right|_{D}\right) \supset F_{2}\left(\left.G\right|_{D}\right) \supset \cdots \supset F_{r}\left(\left.G\right|_{D}\right) \supset F_{r+1}\left(\left.G\right|_{D}\right)=0 .
$$

together with weights $\left\{a_{i}(G)\right\}$. A morphism of parabolic sheaves $G \rightarrow E$ is a morphism of sheaves $g: G \rightarrow E$ such that whenever $a_{i}(G)>a_{j}(E)$, then $\left.g\right|_{D}\left(F_{i}\left(\left.G\right|_{D}\right)\right) \subset F_{j+1}\left(\left.E\right|_{D}\right)$.

Consider now a subsheaf $F \hookrightarrow E$ such that the quotient $Q=E / F$ is torsion-free. In this case, $\left.\left.F\right|_{D} \rightarrow E\right|_{D}$ is an injection (cf. [2, $\left.\S 1.8\right]$ ). Then we have the following induced parabolic structure on the subsheaf $F$.

Definition 2.3. [2, $\S 1.8]$ Let $e:\left.\left.F\right|_{D} \hookrightarrow E\right|_{D}$ denote the inclusion. We define a quasiparabolic structure on $F$ along $D$ by pulling back the flag $\mathfrak{F}_{D}$ by $e$.

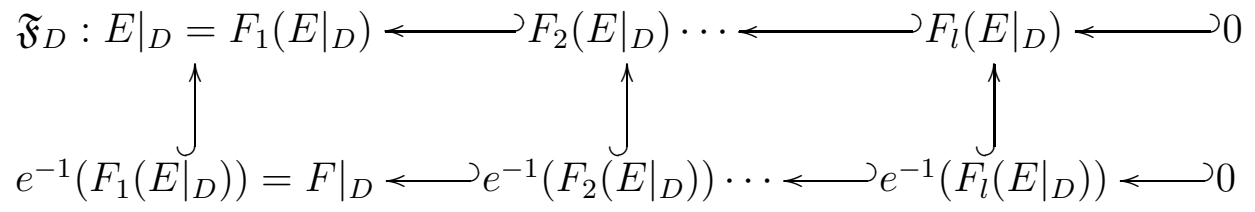

Define the flag $\mathfrak{F}_{D}^{\prime}$ of $\left.F\right|_{D}$ by choosing the irredundant terms of the filtration and call them $F_{j}\left(\left.F\right|_{D}\right)$ where $j \in J \subset\{1,2, \ldots, l\}$. The weight $a_{j}(F)$ associated to $F_{j}\left(\left.F\right|_{D}\right)$ is determined as follows. If $i$ is the largest integer such that $F_{j}\left(\left.F\right|_{D}\right)=e^{-1}\left(F_{i}\left(\left.E\right|_{D}\right)\right)$, then define $a_{j}(F):=a_{i}(E)$. This parabolic structure on $F$ is called the induced parabolic structure.

With this definition, the inclusion $F \hookrightarrow E$ is in fact a morphism of parabolic sheaves (as in Definition 2.2). Hence $F$ is parabolic subsheaf of $E$. The induced structure on $F$ is the "maximum parabolic structure" making the inclusion morphism a morphism of parabolic sheaves. We next define the concepts of parabolic slope and parabolic stability. Let $L$ be an ample line bundle on $X$ with $\operatorname{dim} X=n$.

Definition 2.4. The parabolic weight of $E$ denoted by $\mu$-wt $(E)$ is defined as:

$$
\mu-\mathrm{wt}(E)=\sum_{i=1}^{l} a_{i}(E)\left[r\left(F_{i}\left(\left.E\right|_{D}\right)\right)-r\left(F_{i+1}\left(\left.E\right|_{D}\right)\right)\right] D \cdot L^{n-1} .
$$

Here $r\left(F_{i}\left(\left.E\right|_{D}\right)\right)$ denotes the rank of $F_{i}\left(\left.E\right|_{D}\right)$.

The motivation for the definition is the following. Let $i: D \hookrightarrow X$ denote the inclusion. Then $c_{1}\left(i_{*} F_{i}\left(\left.E\right|_{D}\right)\right)=r\left(F_{i}\left(\left.E\right|_{D}\right)\right) D$, cf. [2, Lemma 2.2]. Thus the above definition is the same as:

$$
\mu-\mathrm{wt}(E)=\sum_{i=1}^{l} a_{i}(E)\left[c_{1}\left(i_{*} F_{i}\left(\left.E\right|_{D}\right)\right) \cdot L^{n-1}-c_{1}\left(i_{*} F_{i+1}\left(\left.E\right|_{D}\right)\right) \cdot L^{n-1}\right] .
$$

Definition 2.5. The parabolic slope of $E$ with respect to $L$ is:

$$
\operatorname{par} \mu_{L}(E)=\frac{c_{1}(E) \cdot L^{n-1}+\mu-\mathrm{wt}(E)}{\operatorname{rank} E} .
$$


Definition 2.6. Let $E$ be a parabolic sheaf as above. Then $E$ is said to be parabolic $\mu_{L}$-semistable (resp. parabolic $\mu_{L}$-stable) if for every subsheaf $F$ of $E$ with torsion-free quotient such that $0<\operatorname{rank} F<\operatorname{rank} E$ equipped with the induced parabolic structure, one has the inequality of slopes, $\operatorname{par} \mu_{L}(F) \leq \operatorname{par} \mu_{L}(E)\left(\operatorname{resp} \cdot \operatorname{par} \mu_{L}(F)<\operatorname{par} \mu_{L}(E)\right)$.

2.2. Orbifold sheaves. Let $Y$ be a connected smooth projective variety of dimension $n$. Let $\operatorname{Aut}(Y)$ denote the group of algebraic automorphisms of $Y$. Let $G$ be a finite group and $\rho: G \rightarrow \operatorname{Aut}(Y)$ be an injective group homomorphism.

Definition 2.7. An orbifold sheaf on $Y$ is a coherent torsion-free sheaf $E$ on $Y$ together with a lift of the action of $G$ to $E$. That is, $G$ acts on the total space of stalks of $E$ and the automorphism of the space of stalks for the action of any $g \in G$ is a coherent sheaf isomorphism between $E$ and $\rho\left(g^{-1}\right)^{*} E$. If $E$ is locally free, then $E$ is called an orbifold bundle.

A subsheaf $F$ of an orbifold sheaf $E$ with torsion-free quotient $E / F$ is said to be $G$ saturated if $F$ is left invariant by the action of $G$. Hence $F$ has an induced orbifold sheaf structure. Let $\widetilde{L} \in \operatorname{Pic}(Y)$ be an ample line bundle which is also an orbifold bundle. Recall that the usual Mumford-Takemoto slope of $E$ with respect to $\widetilde{L}$ is $\mu_{\widetilde{L}}(E)=\frac{c_{1}(E) \cdot \widetilde{L}^{\operatorname{dim} Y-1}}{\operatorname{rank} E}$.

Definition 2.8. An orbifold sheaf $E$ is said to be orbifold semistable (resp. orbifold stable) if for all $G$-saturated subsheaves $F$ of $E$ such that $0<\operatorname{rank} F<\operatorname{rank} E$ one has $\mu_{\widetilde{L}}(F) \leq \mu_{\widetilde{L}}(E)\left(\operatorname{resp} . \mu_{\widetilde{L}}(F)<\mu_{\widetilde{L}}(E)\right)$.

Note that if $E$ is an orbifold sheaf which is $\mu_{\widetilde{L}^{-}}$(semi)stable, then clearly $E$ is orbifold (semi)stable. There is a converse statement in case of semistability which is as follows.

Proposition 2.9. [3, Lemma 2.7] An orbifold bundle is orbifold semistable if and only if it is semistable in the sense of Mumford-Takemoto.

Let $p: Y \rightarrow Y / G=: X$ be the projection. Assume that the quotient $X$ is a smooth variety. Let $E$ be an orbifold sheaf on $Y$. The direct image sheaf $p_{*} E$ on $X$ naturally has a $G$-action compatible with the trivial action of $G$ on the quotient $X$. Let $\left(p_{*} E\right)^{G}$ denote the subsheaf of $G$-invariants in $p_{*} E$.

Definition 2.10. Let $A v$ denote the averaging Reynolds operator $A v: p_{*} E \rightarrow p_{*} E$ given by $A v=\frac{1}{|G|} \sum_{h \in G} t_{h}$. Here $t_{h}$ is the endomorphism of $p_{*} E$ induced by the action of $h \in G$. The image of $A v$ is the invariant subsheaf $\left(p_{*} E\right)^{G}$. Thus we have a short exact sequence:

$$
0 \rightarrow\left(p_{*} E\right)^{G} \rightarrow p_{*} E \rightarrow \frac{p_{*} E}{\left(p_{*} E\right)^{G}} \rightarrow 0 .
$$

When $E$ is an orbifold bundle, the direct image sheaf $p_{*} E$ is also locally free. In this case, since the above exact sequence splits, the subsheaf of invariants $\left(p_{*} E\right)^{G}$ is locally free as well.

\section{Parabolic structures associated to LM Bundles}

Consider a smooth projective surface $X$ over the field $\mathbb{C}$. For any smooth curve $C \stackrel{i}{\hookrightarrow} X$, consider a complete base-point free $g_{d}^{1}$ on $C$, say $A$ (i.e. $A \in \operatorname{Pic}^{d}(C)$ is base-point free 
with $\left.h^{0}(A)=2\right)$. We then have the following short exact sequence corresponding to the linear series $\left(A, H^{0}(C, A)\right)$ on $C$ :

$$
0 \longrightarrow A^{\vee} \longrightarrow H^{0}(C, A) \otimes \mathcal{O}_{C} \longrightarrow A \longrightarrow 0 \text {. }
$$

The dual LM bundle $F_{C, A}=: F$ associated to $(C, A)$ on $X$ is given by the short exact sequence:

$$
0 \longrightarrow F \longrightarrow H^{0}(C, A) \otimes \mathcal{O}_{X} \longrightarrow i_{*} A \longrightarrow 0 \text {. }
$$

Since $F$ is a rank two vector bundle with determinant $\mathcal{O}_{X}(-C)$, we have $F^{\vee} \simeq F \otimes$ $(\operatorname{det} F)^{\vee} \simeq F \otimes \mathcal{O}_{X}(C)$. Note that $F^{\vee}$ is the LM bundle associated to $(C, A)$ and sits in the following sequence:

$$
0 \longrightarrow H^{0}(C, A)^{\vee} \otimes \mathcal{O}_{X} \longrightarrow F \otimes \mathcal{O}_{X}(C) \longrightarrow i_{*}\left(\left.A^{\vee} \otimes \mathcal{O}_{X}(C)\right|_{C}\right) \longrightarrow 0 .
$$

We now associate parabolic vector bundles to the dual LM bundle $F$ in two ways.

3.1. Parabolic LM bundle. We first associate a parabolic structure to the vector bundle $F$ along the divisor $C$ on $X$. Restrict (2) to the curve $C$ to get the following exact sequence on $C$ :

$$
\left.\left.0 \longrightarrow A \otimes \mathcal{O}_{X}(-C)\right|_{C} \longrightarrow F\right|_{C} \longrightarrow H^{0}(C, A) \otimes \mathcal{O}_{C} \longrightarrow A \longrightarrow 0 .
$$

From the above sequence and (1), we also get

$$
\left.\left.0 \longrightarrow A \otimes \mathcal{O}_{X}(-C)\right|_{C} \longrightarrow F\right|_{C} \longrightarrow A^{\vee} \longrightarrow 0 \text {. }
$$

This gives a quasi-parabolic structure on $F$ along the curve $C$, the flag being given by:

$$
\mathfrak{F}_{C}:\left.\left.F\right|_{C} \supset_{a_{1}} A \otimes \mathcal{O}_{X}(-C)\right|_{C} \supset_{a_{2}} 0 .
$$

Here we have associated weights $0 \leq a_{1}<a_{2}<1$ to get a parabolic vector bundle $\left(F, \mathfrak{F}_{C}, a_{1}, a_{2}\right)$. Let us denote $F$ along with the above parabolic structure by $F_{*}$. We call this parabolic vector bundle the (dual) parabolic LM bundle.

Remark 3.1. Dualizing the exact sequence (4), we get the following exact sequence on $C$, where $E_{C, A}=F^{\vee}$ is the LM bundle:

$$
\left.0 \longrightarrow A \longrightarrow\left(E_{C, A}\right)\right|_{C} \longrightarrow\left(\left.A \otimes \mathcal{O}_{X}(-C)\right|_{C}\right)^{\vee} \longrightarrow 0 \text {. }
$$

This gives a parabolic structure on the LM bundle $E_{C, A}$, by associating weights $0 \leq a_{1}<$ $a_{2}<1$ given by

$$
\left.\left(E_{C, A}\right)\right|_{C} \supset_{a_{1}} A \supset_{a_{2}} 0 .
$$

We call the above parabolic vector bundle the parabolic LM bundle. However, in this paper, we work with the dual parabolic LM bundle $F_{*}$.

3.2. Parabolic Blown up LM bundle. Next, we obtain a parabolic structure on the pullback of $F$ to a blown up surface, along the exceptional divisor. Let $X, C, A$ and $F$ be as in the $\S 3.1$. Consider a point $q$ of multiplicity one on $C$.

Consider the blow up of $X$ at $q$, say $\widetilde{X}$. Let $\pi: \widetilde{X} \rightarrow X$ denote the blow down map, and $E \subset \widetilde{X}$, the exceptional divisor. Pull back the exact sequence (2) by $\pi$ to the blown-up surface $\widetilde{X}$ :

$$
0 \rightarrow \pi^{*} F=: \widetilde{F} \rightarrow H^{0}(C, A) \otimes \mathcal{O}_{\widetilde{X}} \rightarrow \pi^{*} i_{*} A \rightarrow 0
$$


Restricting the above sequence to the exceptional divisor $E$, we get:

$$
\left.0 \rightarrow M \rightarrow \widetilde{F}\right|_{E} \simeq F(q) \otimes \mathcal{O}_{E} \rightarrow H^{0}(C, A) \otimes \mathcal{O}_{E} \rightarrow A(q) \otimes \mathcal{O}_{E} \rightarrow 0 .
$$

Here $M$ is the kernel of the restriction. On $\tilde{X}$, we have the following commutative diagram, where $\widetilde{C}$ is the strict transform of $C$ in $\widetilde{X}$.

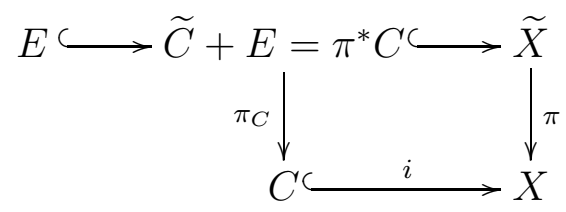

Pulling back the short exact sequence (1) on $C$ to $\pi^{*} C=\widetilde{C}+E$ and restricting to $E$, we get:

$$
0 \rightarrow A^{\vee}(q) \otimes \mathcal{O}_{E} \rightarrow H^{0}(C, A) \otimes \mathcal{O}_{E} \rightarrow A(q) \otimes \mathcal{O}_{E} \rightarrow 0
$$

From the above short exact sequence and from (15), we get:

$$
0 \rightarrow M \rightarrow F(q) \otimes \mathcal{O}_{E} \rightarrow A^{\vee}(q) \otimes \mathcal{O}_{E} \rightarrow 0
$$

The kernel $M$ is in fact a line bundle of the form $M=W \otimes \mathcal{O}_{E}$ where $W$ is a one dimensional vector space. We thus have the following parabolic structure on $\widetilde{F}$ along $E$ by associating weights $0 \leq b_{1}<b_{2}<1$ :

$$
\widetilde{\mathfrak{F}}_{E}:\left.\widetilde{F}\right|_{E}=F(q) \otimes \mathcal{O}_{E} \supset_{b_{1}} M=W \otimes \mathcal{O}_{E} \supset_{b_{2}} 0
$$

We denote the corresponding parabolic vector bundle by $\widetilde{F}_{*}$ and call it the (dual) blown up parabolic LM bundle.

\section{Stability of the PaRABolic BUndLES}

In this section, we discuss the parabolic stability of the parabolic vector bundles $F_{*}$ on $X$ and $\widetilde{F}_{*}$ on $\widetilde{X}$.

4.1. Parabolic stability of $F_{*}$. Consider a rank one coherent subsheaf $G$ of $F$ with torsion-free quotient $F / G$. Then, $G$ is a line bundle, cf. [7, Prop. $1.1 \& 1.9]$. We give the induced parabolic structure on $G$, induced from the parabolic vector bundle $F_{*}$ defined by the parabolic structure $\left.\left.F\right|_{C} \supset_{a_{1}} A \otimes \mathcal{O}_{X}(-C)\right|_{C} \supset_{a_{2}}$. By the procedure explained in Definition [2.3, the induced structure is given as follows.

Lemma 4.1. The induced parabolic structure on $G$ along $C$ is given by

$$
\left.G\right|_{C} \supset_{a_{1}} 0 \text { or }\left.G\right|_{C} \supset_{a_{2}} 0 \text {. }
$$

Proof. Let $e^{\prime}: G \hookrightarrow F$ denote the inclusion. Since $F / G$ is torsion-free, the restriction $\left.e^{\prime}\right|_{C}:\left.\left.G\right|_{C} \hookrightarrow F\right|_{C}$ is also an injection. Denote $e:=\left.e^{\prime}\right|_{C}$. The induced quasi-parabolic structure on $G$ is given as under.

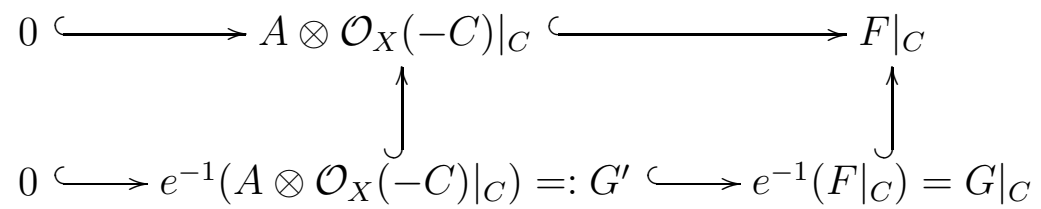


Since $G^{\prime}=e^{-1}\left(\left.A \otimes \mathcal{O}_{X}(-C)\right|_{C}\right)$ is a subsheaf of the line bundle $\left.G\right|_{C}, G^{\prime}$ is either of rank 0 or rank 1.

- If $G^{\prime}$ is of rank 0, then since $\left.G^{\prime} \hookrightarrow A \otimes \mathcal{O}_{X}(-C)\right|_{C}$, it is torsion-free and hence $G^{\prime}=0$. In this case, the parabolic structure on $G$ is given by $\left.G\right|_{C} \supset 0$ with weight $a_{1}$.

- If $G^{\prime}$ is of rank 1 , then the quotient $\frac{\left.G\right|_{C}}{G^{\prime}}$ is of rank 0 , and is a subsheaf of the quotient $\frac{\left.F\right|_{C}}{\left.A \otimes \mathcal{O}_{X}(-C)\right|_{C}} \simeq A^{\vee}$. Hence the quotient is 0 and $\left.G^{\prime} \simeq G\right|_{C}$. In this case, the parabolic structure on $G$ is given by $\left.G\right|_{C} \supset 0$ with weight $a_{2}$.

Hence the induced parabolic structure on $G$ is given by $\left.G\right|_{C} \supset_{a_{i}} 0$ for $i=1,2$.

We now prove Theorem 1.1 regarding the stability of $F_{*}$ with respect to an ample line bundle $L$ on $X$.

Proof of Theorem 1.1. From Definition 2.6, we recall that in order to check parabolic stability of $F_{*}$, it is enough to check the inequality of parabolic slopes for rank one subsheaves $G$ of $F$ with torsion-free quotient equipped with the induced structure. By Lemma 4.1. the induced parabolic structure $G_{*}$ is given by $\left.G\right|_{C} \supset_{a_{i}} 0$ for $i=1,2$.

We know that, $\mu_{L}(F)=\frac{-C \cdot L}{2}$. Since $F$ is $\mu_{L}$-stable, $\mu_{L}(G)<\mu_{L}(F)$. Also $\mu_{L}(G)=$ $c_{1}(G) \cdot L \in \mathbb{Z}$. Thereby,

$$
\mu_{L}(G) \leq \begin{cases}\mu_{L}(F)-1 & \text { if }(C \cdot L) \text { is even } \\ \mu_{L}(F)-\frac{1}{2} & \text { if }(C \cdot L) \text { is odd }\end{cases}
$$

The parabolic weights of $F_{*}$ and $G_{*}$ are given by (cf. Definition 2.4):

$$
\mu \text {-wt }(F)=\left(a_{1}+a_{2}\right)(C \cdot L) \text { and } \mu \text {-wt }(G)=a_{i}(C \cdot L) \text { for } i=1,2 .
$$

Next we compute the parabolic slopes of $F_{*}$ and $G_{*}$.

$$
\begin{gathered}
\operatorname{par} \mu_{L}(F)=\frac{c_{1}(F) \cdot L+\mu-\mathrm{wt}(F)}{2}=\mu_{L}(F)+\frac{\left(a_{1}+a_{2}\right)(C \cdot L)}{2} \text { and, } \\
\operatorname{par} \mu_{L}(G)=\mu_{L}(G)+a_{i}(C \cdot L) \text { where } i=1 \text { or } 2 .
\end{gathered}
$$

Since $a_{1}<a_{2}$, it is enough to prove that $\mu_{L}(G)+a_{2}(C \cdot L)<\operatorname{par} \mu_{L}(F)$ in order to show that $F_{*}$ is parabolic $\mu_{L}$-stable.

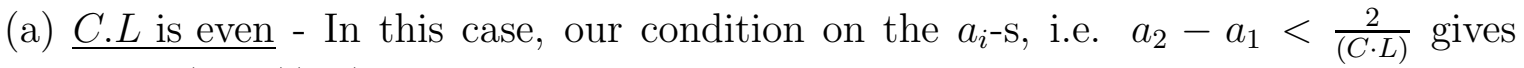
$-1<\frac{\left(a_{1}-a_{2}\right)(C \cdot L)}{2}$. Thus,

$$
\begin{aligned}
\mu_{L}(G)+a_{2}(C \cdot L) & \leq \mu_{L}(F)-1+a_{2}(C \cdot L) \\
& <\mu_{L}(F)+\frac{\left(a_{1}+a_{2}\right)(C \cdot L)}{2}=\operatorname{par} \mu_{L}(F) .
\end{aligned}
$$

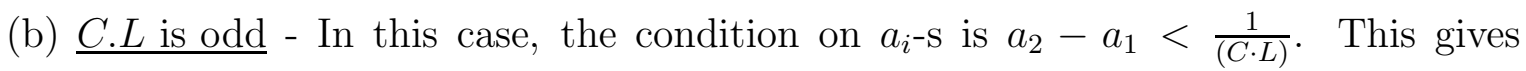
$-\frac{1}{2}<\frac{\left(a_{1}-a_{2}\right)(C \cdot L)}{2}$. Thus,

$$
\begin{aligned}
\mu_{L}(G)+a_{2}(C \cdot L) & \leq \mu_{L}(F)-\frac{1}{2}+a_{2}(C \cdot L) \\
& <\mu_{L}(F)+\frac{\left(a_{1}+a_{2}\right)(C \cdot L)}{2}=\operatorname{par} \mu_{L}(F) .
\end{aligned}
$$


By parts (a) and (b), $F_{*}$ is parabolic $\mu_{L}$-stable.

Corollary 4.2. Let $X$ be a smooth, projective $K 3$ surface and $L \in \operatorname{Pic}(X)$ an ample line bundle such that a general curve $C \in|L|$ has genus $g$, Clifford dimension 1 and maximal gonality $k$. Let the Brill-Noether number $\rho(g, 1, d)>0$. For a general $C \in|L|$, consider the rank 2 dual LM bundle $F:=F_{C, A}$ associated with a general complete, base-point free $g_{d}^{1}$, say $A$ on $C$. Then the dual Parabolic LM bundle $\left(F, \mathfrak{F}_{C}, a_{1}, a_{2}\right)$ is parabolic $\mu_{L^{-}}$stable, whenever

$$
a_{2}-a_{1}<\frac{1}{g-1}
$$

Proof. Lelli-Chiesa [13, Theorem 4.3] proves that such an $F=F_{C, A}$ is $\mu_{L^{-}}$-stable. The proof follows from Theorem 1.1 by observing that on a K3 surface $C \cdot L=\left(C^{2}\right)=2 g-2$, which is even.

Corollary 4.3. Let $X=\mathbb{P}_{\mathbb{C}}^{2}$, the projective plane. Consider $\mathcal{O}(d)$ for an odd $d>0$. Let $C \in|\mathcal{O}(d)|$ be a general smooth curve, and $A=\left.\mathcal{O}(r d)\right|_{C}$ (for $r>0$ ) on $C$. Let $V \subset H^{0}(C, A)$ be a general two dimensional subspace of global sections. Consider the dual $L M$ bundle $F:=F_{C, A, V}$ associated to the triple $(C, A, V)$, and the associated parabolic structure on $F$, say $\left(F, \mathfrak{F}_{C}, a_{1}, a_{2}\right)$ where $a_{2}-a_{1}<\frac{1}{d}$. Then $\left(F, \mathfrak{F}_{C}, a_{1}, a_{2}\right)$ is parabolic $\mu_{\mathcal{O}(1)}$-stable.

Proof. From [19, Theorem 1.2, Remark 4.10], we observe that the dual LM bundle $F$ is $\mu_{\mathcal{O}(1)}$-stable. Since $L=\mathcal{O}(1)$, for $C \in|\mathcal{O}(d)|$, we get $C \cdot L=d$ (which is odd in our case). The result thus follows.

4.2. Parabolic stability of $\widetilde{F}_{*}$. The line bundle $\pi^{*} L$ is not ample on $\widetilde{X}$ because it is trivial when restricted to the exceptional divisor. Consider the line bundle $L_{n}$ on $\tilde{X}$ which is ample (by [4, Lemma 3]) for $n$ sufficiently large,

$$
L_{n}=n \pi^{*} L \otimes \mathcal{O}_{\widetilde{X}}(-E) .
$$

We study the parabolic stability of $\widetilde{F}_{*}$ with respect to the ample line bundle $L_{n}$. We first note the following about the $\mu_{L_{n}}$-stability of $\widetilde{F}$ in the following Remark.

Remark 4.4. If $F$ is a $\mu_{L}$-stable vector bundle on $X$, then there exists an $n_{0} \in \mathbb{Z}$ such that for all $n \geq n_{0}$, the vector bundle $\widetilde{F}=\pi^{*} F$ is a $\mu_{L_{n}}$-stable vector bundle on $\widetilde{X}$. Refer [17. Prop. $2.2(1)]$ for a proof.

Consider a rank 1 coherent subsheaf $\widetilde{G}$ of $\widetilde{F}$ with torsion-free quotient. By similar arguments as before, $\widetilde{G}$ is a line bundle. We give the induced parabolic structure on $\widetilde{G}$ induced from $\widetilde{F}_{*}$ which is defined by $\left.\widetilde{F}\right|_{E} \supset_{b_{1}} M=W \otimes \mathcal{O}_{E} \supset_{b_{2}} 0$.

Lemma 4.5. The induced parabolic structure on $\widetilde{G}$ along $E$ is given either by

$$
\left.\widetilde{G}\right|_{E} \supset_{b_{1}} 0 \text { or }\left.\widetilde{G}\right|_{E} \supset_{b_{2}} 0 \text {. }
$$

Proof. The proof is exactly on the same lines as the proof of Lemma 4.1.

We now prove Theorem 1.2 about the $\mu_{L_{n}}$-stability of $\widetilde{F}_{*}$ for $n \geq n_{0}$, where $n_{0}$ is as in Remark 4.4). 
Proof of Theorem 1.2. Since the dual LM bundle $F$ is $\mu_{L}$-stable, by Remark 4.4, there is an integer $n_{0}$ such that for all $n \geq n_{0}, \widetilde{F}$ is $\mu_{L_{n}}$-stable. Note that,

$$
\mu_{L_{n}}(\widetilde{F})=\frac{c_{1}(\widetilde{F}) \cdot L_{n}}{2} \simeq \frac{\pi^{*} \mathcal{O}_{X}(-C) \cdot\left(n \pi^{*} L-E\right)}{2}=\frac{-n(C \cdot L)}{2} .
$$

If $\widetilde{G}$ is any rank 1 subsheaf of $\widetilde{F}$ with torsion-free quotient, then $\mu_{L_{n}}(\widetilde{G})<\mu_{L_{n}}(\widetilde{F})$. Since $\mu_{L_{n}}(\widetilde{G})=c_{1}(\widetilde{G}) \cdot L_{n} \in \mathbb{Z}$, we get:

$$
\mu_{L_{n}}(\widetilde{G}) \leq \begin{cases}\mu_{L_{n}}(\widetilde{F})-\frac{1}{2} & \text { if }(C \cdot L) \text { and } n \text { are odd } \\ \mu_{L_{n}}(\widetilde{F})-1 & \text { otherwise. }\end{cases}
$$

As discussed in the previous theorem, in order to check for parabolic stability it is enough to check the slope inequality for rank 1 subsheaves $\widetilde{G} \subset \widetilde{F}$ with torsion-free quotient equipped with the induced parabolic structure. The induced parabolic structure on such a $\widetilde{G}$ is $\left.\widetilde{G}\right|_{E} \supset_{b_{i}} 0$ for $i=1,2$. So the parabolic weights of $\widetilde{F}$ and $\widetilde{G}$ are:

$$
\mu-\mathrm{wt}(\widetilde{F})=\left(b_{1}+b_{2}\right) E \cdot\left(n \pi^{*} L-E\right)=-\left(b_{1}+b_{2}\right) E^{2} .
$$

Since $\left(E^{2}\right)=-1$, we get $\mu$-wt $(\widetilde{F})=b_{1}+b_{2}$. Similarly, we get that $\mu$-wt $(\widetilde{G})=b_{i} E \cdot L_{n}=b_{i}$. We now compute the parabolic slopes,

$$
\begin{gathered}
\operatorname{par} \mu_{L_{n}}(\widetilde{G})=\mu_{L_{n}}(\widetilde{G})+\mu-\mathrm{wt}(\widetilde{G})=\mu_{L_{n}}(\widetilde{G})+b_{i} . \\
\operatorname{par} \mu_{L_{n}}(\widetilde{F})=\mu_{L_{n}}(\widetilde{F})+\frac{\mu-\mathrm{wt}(\widetilde{F})}{2}=\mu_{L_{n}}(\widetilde{F})+\frac{b_{1}+b_{2}}{2} .
\end{gathered}
$$

Since $b_{1}<b_{2}$, it is enough to check that $\mu_{L_{n}}(\widetilde{G})+b_{2}<\operatorname{par} \mu_{L_{n}}(\widetilde{F})$. Since $0 \leq b_{1}<b_{2}<$ 1 , we have $b_{2}-b_{1}<1$. Again we have two cases.

(a) Both $n$ and $(C \cdot L)$ are odd - Note that, $-\frac{1}{2}<\frac{b_{1}-b_{2}}{2}$. We now have

$$
\begin{aligned}
\mu_{L_{n}}(\widetilde{G})+b_{2} & \leq \mu_{L_{n}}(\widetilde{F})-\frac{1}{2}+b_{2} \\
& <\mu_{L_{n}}(\widetilde{F})+\frac{b_{1}+b_{2}}{2}=\operatorname{par} \mu_{L_{n}}(\widetilde{F}) .
\end{aligned}
$$

(b) At least one of $n$ or $(C \cdot L)$ is even - Note that $\frac{b_{2}-b_{1}}{2}<b_{2}-b_{1}<1$, which gives $-1<\frac{b_{1}-b_{2}}{2}$. We have

$$
\begin{aligned}
\mu_{L_{n}}(\widetilde{G})+b_{2} & \leq \mu_{L_{n}}(\widetilde{F})-1+b_{2} \\
& <\mu_{L_{n}}(\widetilde{F})+\frac{b_{1}+b_{2}}{2}=\operatorname{par} \mu_{L_{n}}(\widetilde{F}) .
\end{aligned}
$$

Thus from parts (a) and (b), we have that the (dual) parabolic blown up LM bundle $\widetilde{F}_{*}$ is parabolic $\mu_{L_{n}}$-stable for all weights $b_{1}$ and $b_{2}$ and for $n \geq n_{0}$, where $n_{0}$ is as in Remark 4.4.

Corollary 4.6. Let $X$ be a smooth, projective $K 3$ surface and $L \in \operatorname{Pic}(X)$ an ample line bundle such that a general curve $C \in|L|$ has genus $g$, Clifford dimension 1 and maximal gonality $k$. Let the Brill Noether number $\rho(g, 1, d)>0$. For a general $C \in|L|$, consider the rank 2 dual LM bundle $F:=F_{C, A}$ associated with a general complete, base-point free $g_{d}^{1}$ say $A$ on $C$. Then, there is an integer $n_{0}$ such that the dual parabolic blown up LM bundle $\left(\widetilde{F}, \widetilde{\mathfrak{F}}_{E}, b_{1}, b_{2}\right)$ is parabolic $\mu_{L_{n}}$-stable, for all choices of weights and for all $n \geq n_{0}$. 
Proof. This result again follows from Lelli-Chiesa [13, Theorem 4.3], whereby such an $F_{C, A}$ is $\mu_{L}$-stable.

Corollary 4.7. Let $X=\mathbb{P}_{\mathbb{C}}^{2}$, the projective plane. Consider $\mathcal{O}(d)$ for an odd number $d>0$. Let $C \in|\mathcal{O}(d)|$ be a general smooth curve, and $A=\left.\mathcal{O}_{X}(r d)\right|_{C}$ for $r>0$ on $C$. Let $V \subset H^{0}(C, A)$ be a general two dimensional subspace of global sections. Consider the dual $L M$ bundle $F:=F_{C, A, V}$ associated to the triple $(C, A, V)$, and the associated parabolic structure on $\widetilde{F}$, say $\left(\widetilde{F}, \widetilde{\mathfrak{F}}_{E}, b_{1}, b_{2}\right)$. Then there is an integer $n_{0}$ such that $\left(\widetilde{F}, \widetilde{\mathfrak{F}}_{E}, b_{1}, b_{2}\right)$ is parabolic $\mu_{L_{n}}$-stable, where $L=\mathcal{O}(1)$ and for all $n \geq n_{0}$.

Proof. The result follows from [19, Theorem 1.2] which proves that the dual LM bundle $F_{C, A, V}$ is $\mu_{\mathcal{O}(1) \text {-stable. }}$

\section{ORBIFOLD LM BUNDLE}

Recall that the quasi-parabolic structure on the parabolic vector bundle $F_{*}$ is given by

$$
\left.F\right|_{C}=\left.F_{1}\left(\left.F\right|_{C}\right) \supset A \otimes \mathcal{O}_{X}(-C)\right|_{C}=F_{2}\left(\left.F\right|_{C}\right) \supset 0=F_{3}\left(\left.F\right|_{C}\right) .
$$

By [14, Definition 1.1], the quasi-parabolic structure can be alternatively described by a filtration of the vector bundle $F$ itself of the form

$$
F=F_{1}(F) \supset F_{2}(F) \supset F_{3}(F)=F(-C) .
$$

The elements of the two filtrations are related as follows (cf. [3, $\S 3]$ ):

$$
F_{i}\left(\left.F\right|_{C}\right)=\frac{F_{i}(F)}{F(-C)} .
$$

We obtain the equivalent formulation of the quasi-parabolic structure on $F_{*}$ from the following lemma.

Lemma 5.1. The quasi-parabolic structure on the (dual) parabolic LM bundle $F_{*}$ is equivalent to the quasi-parabolic structure given by the following filtration of $F$ :

$$
\mathfrak{F}: F \supseteq H^{0}(C, A) \otimes \mathcal{O}_{X}(-C) \supseteq F(-C) \text {. }
$$

Proof. By the discussion above, we just need to determine $F_{2}(F)$. We also have, $F_{2}\left(\left.F\right|_{C}\right)=$ $F_{2}(F) / F(-C)$. Hence $F_{2}(F)$ has to sit in the following short exact sequence:

$$
0 \rightarrow F(-C) \rightarrow F_{2}(F) \longrightarrow i_{*}\left(\left.A \otimes \mathcal{O}_{X}(-C)\right|_{C}\right) \rightarrow 0 .
$$

Tensoring the exact sequence (2) which defines $F$ by $\mathcal{O}_{X}(-C)$, we get

$$
0 \longrightarrow F(-C) \longrightarrow H^{0}(C, A) \otimes \mathcal{O}_{X}(-C) \longrightarrow i_{*}\left(\left.A \otimes \mathcal{O}_{X}(-C)\right|_{C}\right) \longrightarrow 0 .
$$

Thus, $F_{2}(F)=H^{0}(C, A) \otimes \mathcal{O}_{X}(-C)$.

So, the parabolic vector bundle $\left(F, \mathfrak{F}_{C}, a_{1}, a_{2}\right)$ from $\S 3.1$ can alternatively be described as $\left(F, \mathfrak{F}, a_{1}, a_{2}\right)$ where the flag $\mathfrak{F}$ is described in Lemma 5.1.

Remark 5.2. Set $a_{0}=a_{2}-1$ and $a_{3}=1$, where $F_{*}=\left(F, \mathfrak{F}, a_{1}, a_{2}\right)$ is the (dual) parabolic $L M$ bundle. Let $[t]$ denote the integral part of any $t \in \mathbb{R}$. For each $t \in \mathbb{R}$, consider the following locally free sheaves $E_{t}=F_{i}(F)(-[t] C)$, where $a_{i-1}<t-[t] \leq a_{i}$. Note that the locally free sheaves $\left\{E_{t}\right\}$ give a filtration which is decreasing, i.e. if $t \geq t^{\prime}$, then $E_{t} \subseteq E_{t^{\prime}}$. This filtration is left continuous. Further, this filtration has a jump at some $t$, i.e. for all 
$\epsilon>0, E_{t+\epsilon} \neq E_{t}$ if and only if $t-[t]=a_{i}$ where $i=1,2$. Finally, for all $t \in \mathbb{R}$, we have $E_{t+1}=E_{t}(-C)$. This filtration $\left\{E_{t}\right\}_{t \in \mathbb{R}}$ completely determines the parabolic vector bundle $\left(F, \mathfrak{F}, a_{1}, a_{2}\right)$, cf. [14] and [3]. In particular, the parabolic vector bundle $F_{*}$ is completely determined by the filtration of vector bundles

$$
\begin{aligned}
E_{t} & =F \text { for } t \in\left[0, a_{1}\right] \\
& =H^{0}(C, A) \otimes \mathcal{O}_{X}(-C) \text { for } t \in\left(a_{1}, a_{2}\right] \\
& =F(-C) \text { for } t \in\left(a_{2}, 1\right] .
\end{aligned}
$$

We recall the Kawamata covering Theorem [12, Prop. 4.1.12]. Given a positive integer $N>0$, there is a non-singular connected projective variety $Y$ and a finite Galois morphism $p: Y \rightarrow X$ with Galois group $\Gamma=\operatorname{Gal}(\operatorname{Rat}(Y) / \operatorname{Rat}(X))$ such that $p^{*} C$ is a non-reduced divisor of the form $N C^{\prime}$ with $\left(p^{*} C\right)_{\text {red }}=C^{\prime}$. Here $C^{\prime}$ is a smooth curve on $Y$. We then have the following commutative diagram, for any $m$ such that $1 \leq m<N$.

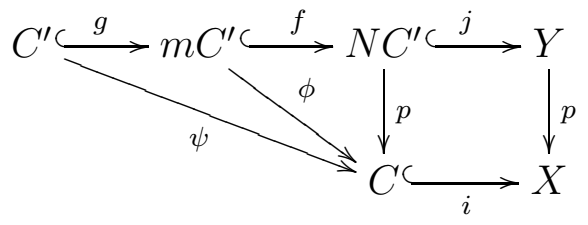

Pulling back the exact sequence (11) to $m C^{\prime}$ by $\phi$, we get:

$$
0 \rightarrow \phi^{*} A^{\vee} \rightarrow H^{0}(C, A) \otimes \mathcal{O}_{m C^{\prime}} \rightarrow \phi^{*} A \rightarrow 0 .
$$

Denote $A^{\prime}=\phi^{*} A$. Then $\left(A^{\prime}, H^{0}(C, A)\right)$ is a base-point free linear system on the curve $m C^{\prime}$ (which is non-reduced when $m>1$ ). We can thus consider the dual LM bundle $F^{\prime}$ on $Y$ associated to the triple $\left(m C^{\prime}, A^{\prime}, H^{0}(C, A)\right)$ given by the following short exact sequence:

$$
0 \rightarrow F^{\prime} \rightarrow H^{0}(C, A) \otimes \mathcal{O}_{Y} \rightarrow j_{*} f_{*} A^{\prime} \rightarrow 0 .
$$

Remark 5.3. The dual $L M$ bundle $F^{\prime}$ on $Y$ associated to the triple $\left(m C^{\prime}, A^{\prime}, H^{0}(C, A)\right)$ has a natural orbifold structure. Indeed, $H^{0}(C, A) \otimes \mathcal{O}_{Y} \rightarrow j_{*} f_{*} A^{\prime}$ is a morphism between orbifold sheaves on $Y$ compatible with the action of $\Gamma$ on both the sheaves. We call this orbifold bundle $F^{\prime}$ on $Y$ "the orbifold LM bundle".

By the construction of Biswas [3, $\S 2 \mathrm{c}]$, this orbifold LM bundle corresponds to a uniquely determined parabolic bundle on $X$. We now determine this parabolic vector bundle. For any $t \in \mathbb{R}$, define, as in [3],

$$
E_{t}=\left(p_{*}\left(F^{\prime} \otimes \mathcal{O}_{Y}\left([-t \cdot N] C^{\prime}\right)\right)\right)^{\Gamma}
$$

Just as in Remark [5.2, this defines a parabolic sheaf on $X$, cf. [3, $\S 2$ ] and [14, $\S 1]$. We first have the following preliminary lemma.

Lemma 5.4. Let $A$ be a line bundle on $C$. For any $m$ such that $1 \leq m<N$, consider the line bundle $L^{\prime}:=\left.\psi^{*} A \otimes \mathcal{O}_{Y}\left(-m C^{\prime}\right)\right|_{C^{\prime}}$ on $C^{\prime}$. Then, with notations as in the commutative diagram below, the invariant sheaf $\left(i_{*} \psi_{*} L^{\prime}\right)^{\Gamma}=\left(p_{*} j_{*} f_{*}^{\prime} h_{*}^{\prime} g_{*} L^{\prime}\right)^{\Gamma}=0$. 


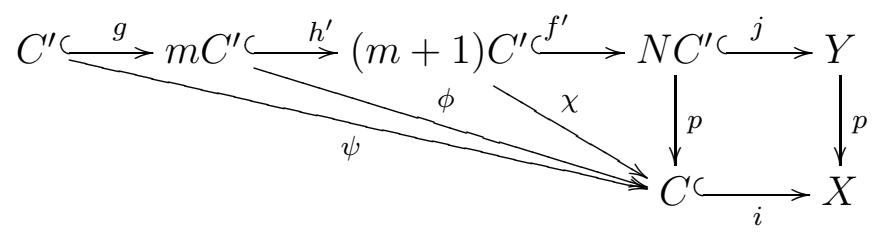

Proof. Corresponding to the closed immersion $h^{\prime}: m C^{\prime} \hookrightarrow(m+1) C^{\prime}$, for $m=1,2, \cdots N-$ 1 , we have the surjective morphism of sheaves $\mathcal{O}_{(m+1) C^{\prime}} \rightarrow h_{*}^{\prime} \mathcal{O}_{m C^{\prime}}$. The ideal sheaf of this closed subscheme is $\left.h_{*}^{\prime} g_{*} \mathcal{O}_{Y}\left(-m C^{\prime}\right)\right|_{C^{\prime}}$. This gives,

$$
\left.0 \rightarrow h_{*}^{\prime} g_{*} \mathcal{O}_{Y}\left(-m C^{\prime}\right)\right|_{C^{\prime}} \rightarrow \mathcal{O}_{(m+1) C^{\prime}} \rightarrow h_{*}^{\prime} \mathcal{O}_{m C^{\prime}} \rightarrow 0
$$

Tensor by $\chi^{*} A$ (and use projection formula) to get

$$
0 \rightarrow h_{*}^{\prime} g_{*}\left(\left.\psi^{*} A \otimes \mathcal{O}_{Y}\left(-m C^{\prime}\right)\right|_{C^{\prime}}\right) \simeq h_{*}^{\prime} g_{*} L^{\prime} \rightarrow \chi^{*} A \rightarrow h_{*}^{\prime}\left(\phi^{*} A\right) \rightarrow 0 .
$$

Pushforward this sequence to $X$ by applying $p_{*} j_{*} f_{*}^{\prime}$ :

$$
0 \rightarrow p_{*} j_{*} f_{*}^{\prime} h_{*}^{\prime} g_{*} L^{\prime} \rightarrow p_{*} j_{*} f_{*}^{\prime} \chi^{*} A \rightarrow p_{*} j_{*} f_{*}^{\prime} h_{*}^{\prime} \phi^{*} A \rightarrow 0 .
$$

That is,

$$
0 \longrightarrow i_{*} \psi_{*} L^{\prime} \longrightarrow i_{*} \chi_{*} \chi^{*} A \longrightarrow i_{*} \phi_{*} \phi^{*} A \longrightarrow 0 \text {. }
$$

Note that the above is a sequence of pushforwards to $X$ by $p$ of $\Gamma$-sheaves from $Y$. Therefore, we can take $\Gamma$-invariants of each of these sheaves. The sequence of $\Gamma$-invariant sheaves continues to be exact, cf. [10, §1.4.3]:

$$
0 \longrightarrow\left(i_{*} \psi_{*} L^{\prime}\right)^{\Gamma} \longrightarrow\left(i_{*} \chi_{*} \chi^{*} A\right)^{\Gamma} \longrightarrow\left(i_{*} \phi_{*} \phi^{*} A\right)^{\Gamma} \longrightarrow 0
$$

However, $\left(i_{*} \chi_{*} \chi^{*} A\right)^{\Gamma} \simeq i_{*} A \simeq\left(i_{*} \phi_{*} \phi^{*} A\right)^{\Gamma}$. Thus, $\left(i_{*} \psi_{*} L^{\prime}\right)^{\Gamma}=0$.

We now prove Theorem 1.3 stated in the introduction.

Proof of Theorem 1.3. We need to prove that the orbifold bundle on $Y$ corresponding to the dual parabolic LM bundle $\left(F, \mathfrak{F}_{C}, 0, \frac{N-m}{N}\right)$ on $X$ is the dual LM bundle $F^{\prime}$. We do that by showing that the parabolic bundle on $X$ corresponding to $F^{\prime}$ is the required one.

We recall the commutative diagram where $1 \leq m<N$ :

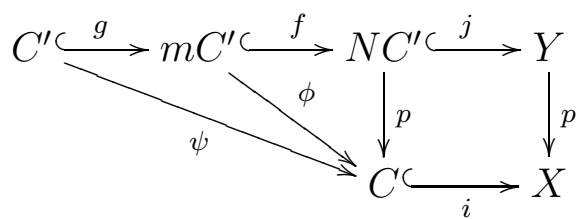

For all $a=1,2, \ldots, N$, we have the following exact sequence which we will use repeatedly in the proof:

$$
0 \rightarrow F^{\prime}\left(-a C^{\prime}\right) \rightarrow F^{\prime}\left(-(a-1) C^{\prime}\right) \rightarrow j_{*} f_{*} g_{*}\left(\left.F^{\prime}\left(-(a-1) C^{\prime}\right)\right|_{C^{\prime}}\right) \rightarrow 0 .
$$

Note that, just as in the short exact sequence (41), $\left.F^{\prime}\right|_{m C^{\prime}}$ fits in the following short exact sequence of sheaves on $m C^{\prime}$ :

$$
\left.\left.0 \longrightarrow \phi^{*} A \otimes \mathcal{O}_{Y}\left(-m C^{\prime}\right)\right|_{m C^{\prime}} \longrightarrow F^{\prime}\right|_{m C^{\prime}} \longrightarrow \phi^{*} A^{\vee} \longrightarrow 0 .
$$

We restrict this to $C^{\prime}$ :

$$
\left.\left.0 \longrightarrow \psi^{*} A \otimes \mathcal{O}_{Y}\left(-m C^{\prime}\right)\right|_{C^{\prime}} \longrightarrow F^{\prime}\right|_{C^{\prime}} \longrightarrow \psi^{*} A^{\vee} \longrightarrow 0 \text {. }
$$


By equation (17), we have $E_{0}=\left(p_{*} F^{\prime}\right)^{\Gamma}$. We further have that, if $t \in\left(\frac{a-1}{N}, \frac{a}{N}\right]$ where $a=1,2, \cdots, N-1, N$, then,

$$
E_{t}=\left(p_{*}\left(F^{\prime} \otimes \mathcal{O}_{Y}\left(-a C^{\prime}\right)\right)\right)^{\Gamma} .
$$

We now compute $E_{t}$ as we vary $t \in[0,1]$, and this filtration of sheaves will completely determine the parabolic bundle on $X$ corresponding to $F^{\prime}$.

1. $\underline{t=0}$

Pushforward the exact sequence (6) to $X$ and consider the sequence of $\Gamma$-invariant sheaves (Taking $\Gamma$-invariants is an exact functor). Since $p_{*} j_{*} f_{*} A^{\prime} \simeq i_{*} \phi_{*} \phi^{*} A$, we get

$$
0 \rightarrow\left(p_{*} F^{\prime}\right)^{\Gamma} \rightarrow H^{0}(C, A) \otimes\left(p_{*} \mathcal{O}_{Y}\right)^{\Gamma} \rightarrow\left(i_{*} \phi_{*} \phi^{*} A\right)^{\Gamma} \rightarrow 0 .
$$

Thus we get,

$$
0 \rightarrow\left(p_{*} F^{\prime}\right)^{\Gamma} \rightarrow H^{0}(C, A) \otimes \mathcal{O}_{X} \rightarrow i_{*} A \rightarrow 0 .
$$

Therefore $\left(p_{*} F^{\prime}\right)^{\Gamma}=E_{0}=F$.

2. $t \in\left(0, \frac{N-m}{N}\right]$

Consider $t \in\left(\frac{a-1}{N}, \frac{a}{N}\right]$ for $a=1,2, \ldots, N-m$. Then, $E_{t}=\left(p_{*}\left(F^{\prime} \otimes \mathcal{O}_{Y}\left(-a C^{\prime}\right)\right)\right)^{\Gamma}$. We consider the pushforward of the sequence (8) to $X$ by $p$, noting that $p_{*} j_{*} f_{*} g_{*}\left(F^{\prime}(-(a-\right.$ 1) $\left.\left.C^{\prime}\right)\left.\right|_{C^{\prime}}\right) \simeq i_{*} \psi_{*}\left(\left.F^{\prime}\left(-(a-1) C^{\prime}\right)\right|_{C^{\prime}}\right)$, to get:

$$
\left.0 \rightarrow p_{*} F^{\prime}\left(-a C^{\prime}\right) \rightarrow p_{*} F^{\prime}\left(-(a-1) C^{\prime}\right) \rightarrow i_{*} \psi_{*} F^{\prime}\left(-(a-1) C^{\prime}\right)\right|_{C^{\prime}} \rightarrow 0 .
$$

Now the sheaf $i_{*} \psi_{*}\left(\left.F^{\prime}\left(-(a-1) C^{\prime}\right)\right|_{C^{\prime}}\right)$ fits in the following short exact sequence that we get from (9):

$$
\begin{aligned}
0 \rightarrow i_{*} \psi_{*}\left(\left.\psi^{*} A \otimes \mathcal{O}_{Y}\left((-(a-1)-m) C^{\prime}\right)\right|_{C^{\prime}}\right) & \left.\rightarrow i_{*} \psi_{*} F^{\prime}\left(-(a-1) C^{\prime}\right)\right|_{C^{\prime}} \\
& \rightarrow i_{*} \psi_{*}\left(\left.\psi^{*} A^{\vee} \otimes \mathcal{O}_{Y}\left(-(a-1) C^{\prime}\right)\right|_{C^{\prime}}\right) \rightarrow 0
\end{aligned}
$$

For $a=2, \ldots, N-m$, we have $\left(i_{*} \psi_{*}\left(\left.\psi^{*} A \otimes \mathcal{O}_{Y}\left((-(a-1)-m) C^{\prime}\right)\right|_{C^{\prime}}\right)\right)^{\Gamma}=0$ and $\left(i_{*} \psi_{*}\left(\left.\psi^{*} A^{\vee} \otimes \mathcal{O}_{Y}\left(-(a-1) C^{\prime}\right)\right|_{C^{\prime}}\right)\right)^{\Gamma}=0$ by Lemma 5.4. Hence, $\left(\left.i_{*} \psi_{*} F^{\prime}\left(-(a-1) C^{\prime}\right)\right|_{C^{\prime}}\right)^{\Gamma}=$ 0 and thus $\left(p_{*} F^{\prime}\left(-a C^{\prime}\right)\right)^{\Gamma}=\left(p_{*} F^{\prime}\left(-(a-1) C^{\prime}\right)\right)^{\Gamma}$ for $a=2,3, \cdots, N-m$.

Now it is sufficient to compute $\left(p_{*} F^{\prime}\left(-C^{\prime}\right)\right)^{\Gamma}$ which is in fact $E_{t}$ for $t \in\left(0, \frac{1}{N}\right]$ i.e. the case $a=1$ above. Set $a=1$ in (8) and pushforward the sequence of $\Gamma$-sheaves on $Y$ to $X$ :

$$
0 \rightarrow p_{*} F^{\prime}\left(-C^{\prime}\right) \rightarrow p_{*} F^{\prime} \rightarrow p_{*} j_{*} f_{*} g_{*}\left(\left.F^{\prime}\right|_{C^{\prime}}\right) \simeq i_{*} \psi_{*}\left(\left.F^{\prime}\right|_{C^{\prime}}\right) \rightarrow 0 .
$$

Next, setting $a=1$ in the short exact sequence (10), we get:

$$
\left.0 \rightarrow i_{*} \psi_{*}\left(\left.\psi^{*} A \otimes \mathcal{O}_{Y}\left(-m C^{\prime}\right)\right|_{C^{\prime}}\right) \rightarrow i_{*} \psi_{*} F^{\prime}\right|_{C^{\prime}} \rightarrow i_{*} \psi_{*} \psi^{*} A^{\vee} \rightarrow 0 \text {. }
$$

By Lemma 5.4. $\left(i_{*} \psi_{*}\left(\left.\psi^{*} A \otimes \mathcal{O}_{Y}\left(-m C^{\prime}\right)\right|_{C^{\prime}}\right)\right)^{\Gamma}=0$ since $m<N$. Also, we have $\left(i_{*} \psi_{*} \psi^{*} A^{\vee}\right)^{\Gamma}=$ $i_{*} A^{\vee}$. Hence, $\left(\left.i_{*} \psi_{*} F^{\prime}\right|_{C^{\prime}}\right)^{\Gamma} \simeq i_{*} A^{\vee}$. Considering the invariants of the short exact sequence (11), we get

$$
0 \rightarrow\left(p_{*} F^{\prime}\left(-C^{\prime}\right)\right)^{\Gamma} \rightarrow F \rightarrow i_{*} A^{\vee} \rightarrow 0
$$


From the short exact sequence (3), it follows that $\left(p_{*} F^{\prime}\left(-C^{\prime}\right)\right)^{\Gamma} \simeq H^{0}(C, A) \otimes \mathcal{O}_{X}(-C)$. Thus $E_{t}=H^{0}(C, A) \otimes \mathcal{O}_{X}(-C)$ for $t \in(0,1 / N]$ and $\left(p_{*} F^{\prime}\left(-a C^{\prime}\right)\right)^{\Gamma}=\left(p_{*} F^{\prime}\left(-C^{\prime}\right)\right)^{\Gamma}=$ $H^{0}(C, A) \otimes \mathcal{O}_{X}(-C)$ for $a=2, \ldots, N-m$. Thereby,

$$
E_{t}=H^{0}(C, A) \otimes \mathcal{O}_{X}(-C) \quad \text { for all } \quad t \in\left(0, \frac{N-m}{N}\right] .
$$

3. $t \in\left(\frac{N-m}{N}, \frac{N-m+1}{N}\right]$

In this case, we have $E_{t}=\left(p_{*}\left(F^{\prime} \otimes \mathcal{O}_{Y}\left(-(N-m+1) C^{\prime}\right)\right)\right)^{\Gamma}$. Since $p_{*} j_{*} f_{*} g_{*}\left(F^{\prime}(-(N-\right.$ $\left.\left.m) C^{\prime}\right)\left.\right|_{C^{\prime}}\right)=i_{*} \psi_{*}\left(\left.F^{\prime}\left(-(N-m) C^{\prime}\right)\right|_{C^{\prime}}\right)$, from (8) we have

$$
\begin{aligned}
0 \rightarrow p_{*} F^{\prime}\left(-(N-m+1) C^{\prime}\right) \rightarrow p_{*} F^{\prime}\left(-(N-m) C^{\prime}\right) & \\
& \rightarrow i_{*} \psi_{*}\left(\left.F^{\prime}\left(-(N-m) C^{\prime}\right)\right|_{C^{\prime}}\right) \rightarrow 0
\end{aligned}
$$

From (9), we get

$$
\begin{gathered}
0 \rightarrow\left(i_{*} \psi_{*}\left(\left.\psi^{*} A \otimes \mathcal{O}_{Y}\left(-N C^{\prime}\right)\right|_{C^{\prime}}\right)\right)^{\Gamma} \rightarrow\left(\left.i_{*} \psi_{*} F^{\prime}\left(-(N-m) C^{\prime}\right)\right|_{C^{\prime}}\right)^{\Gamma} \\
\rightarrow\left(i_{*} \psi_{*}\left(\left.\psi^{*} A^{\vee} \otimes \mathcal{O}_{Y}\left(-(N-m) C^{\prime}\right)\right|_{C^{\prime}}\right)\right)^{\Gamma} \rightarrow 0
\end{gathered}
$$

As before, the right hand side term of the above sequence is zero. But note that $\left(i_{*} \psi_{*}\left(\left.\psi^{*} A \otimes \mathcal{O}_{Y}\left(-N C^{\prime}\right)\right|_{C^{\prime}}\right)\right)^{\Gamma}=\left(i_{*} \psi_{*} \psi^{*}\left(\left.A \otimes \mathcal{O}_{X}(-C)\right|_{C}\right)\right)^{\Gamma} \simeq i_{*}\left(\left.A \otimes \mathcal{O}_{X}(-C)\right|_{C}\right)$ and hence $\left(\left.i_{*} \psi_{*} F^{\prime}\left(-(N-m) C^{\prime}\right)\right|_{C^{\prime}}\right)^{\Gamma} \simeq i_{*}\left(\left.A \otimes \mathcal{O}_{X}(-C)\right|_{C}\right)$. Finally, taking $\Gamma$-invariants of (13), we get:

$$
0 \rightarrow\left(p_{*} F^{\prime}\left(-(N-m+1) C^{\prime}\right)\right)^{\Gamma} \rightarrow H^{0}(C, A) \otimes \mathcal{O}_{X}(-C) \rightarrow i_{*}\left(\left.A \otimes \mathcal{O}_{X}(-C)\right|_{C}\right) \rightarrow 0 .
$$

From the exact sequence (2), we get $\left(p_{*} F^{\prime}\left(-(N-m+1) C^{\prime}\right)\right)^{\Gamma} \simeq F \otimes \mathcal{O}_{X}(-C)$. Thus for $t \in\left(\frac{N-m}{N}, \frac{N-m+1}{N}\right]$, we get $E_{t}=F \otimes \mathcal{O}_{X}(-C)=F(-C)$. Since this is the expected end of the flag for the parabolic structure, we deduce, based on the known properties of $E_{t}$ from Biswas [3], that $E_{t}=F(-C)$ for $t \in\left(\frac{N-m}{N}, 1\right]$.

Thus, we have the following sheaves $E_{t}$ for $t \in[0,1]$ :

(1) $E_{0}=F$,

(2) $E_{t}=H^{0}(C, A) \otimes \mathcal{O}_{X}(-C)$ for $t \in\left(0, \frac{N-m}{N}\right]$,

(3) $E_{t}=F(-C)$ for $t \in\left(\frac{N-m}{N}, 1\right]$.

As there is a jump at $t=0$ and at $t=\frac{N-m}{N}$, these give the parabolic weights. Indeed, the parabolic vector bundle on $X$ corresponding to $F^{\prime}$ is:

$$
F \supset_{0} H^{0}(C, A) \otimes \mathcal{O}_{X}(-C) \supset_{\frac{N-m}{N}} F(-C)
$$

or equivalently the $\left(F, \mathfrak{F}_{C}, 0, \frac{N-m}{N}\right)$. This is precisely the description of the required parabolic sheaf.

The above theorem shows that the LM bundles behave well under the parabolic-orbifold bundle correspondence. 


\section{Proof of Theorem 1.4}

We keep notations as in previous sections. Let $L$ be an ample line bundle on $X$.

Corollary 6.1. The dual parabolic LM bundle $\left(F, \mathfrak{F}_{C}, a_{1}, a_{2}\right)$ with $a_{1}=0$ and $a_{2}=\frac{N-m}{N}$ on $X$ is parabolic $\mu_{L}$-semistable if and only if the corresponding Orbifold LM bundle $F^{\prime}$ associated to the triple $\left(m C^{\prime}, \phi^{*} A, H^{0}(C, A)\right)$ on $Y$ is $\mu_{p^{*} L^{-}}$-semistable.

Proof. This is a direct consequence of [3, Lemma 3.13] and the fact that an orbifold bundle

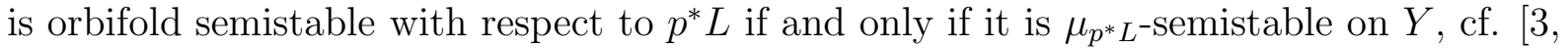
Lemma 2.7].

Consequently, we prove Theorem 1.4 .

Proof of Theorem 1.4. Keep notations as in the statement of the Theorem.

(a) In this case, $X=\mathbb{P}^{2}$ and $C \in|\mathcal{O}(d)|$ is a general smooth degree $d$ curve, where $d$ is odd. Let $A=\left.\mathcal{O}(r d)\right|_{C}$ for any $r \geq 1$, and $V \subset H^{0}(C, A)$ be a general two dimensional subspace. Let $F$ be the dual LM bundle on $X$ corresponding to $(C, A, V)$ which is $\mu_{\mathcal{O}(1)}$-stable, cf. [19, Theorem 1.2]. Given that $m$ is an integer such that $\frac{N-m}{N}<\frac{1}{d}$. Consider the dual parabolic LM bundle $F_{*}=\left(F, \mathfrak{F}_{C}, a_{1}, a_{2}\right)$ where the weights $a_{1}=0$ and $a_{2}=\frac{N-m}{N}<\frac{1}{d}$. By Corollary 4.3, $F_{*}$ is parabolic $\mu_{\mathcal{O}(1)}$-stable. By Corollary 6.1, the dual LM bundle on $Y$ corresponding to the triple $\left(m C^{\prime}, A^{\prime}, V\right)$ on $Y$ is $\mu_{p^{*} \mathcal{O}(1)}$-semistable.

(b) Here $X$ is a smooth projective K3 surface and $L$ is an ample line bundle on $X$ such that a general curve $C \in|L|$ has genus $g$, Clifford dimension 1 and maximal gonality $k$. We have $\rho(g, 1, d)>0$. For a general smooth $C \in|L|$, consider the rank 2 dual LM bundle $F:=F_{C, A}$ associated with a general complete, base-point free $g_{d}^{1}$, say $A$ on $C$. By [13], $F$ is $\mu_{L}$-stable. We have an integer $m$ such that $\frac{N-m}{N}<\frac{1}{g-1}$. Then, by Corollary 4.2, the dual parabolic LM bundle $\left(F, \mathfrak{F}_{C}, a_{1}, a_{2}\right)$ with weights $a_{1}=0$ and $a_{2}=\frac{N-m}{N}<\frac{1}{g-1}$ is parabolic $\mu_{L^{-}}$stable. Then by Corollary 6.1, the LM bundle on $Y$ corresponding to the triple $\left(m C^{\prime}, A^{\prime}, H^{0}(C, A)\right)$ is $\mu_{p^{*} L^{-}}$semistable.

(a') As a particular case of (a), let $C \in|\mathcal{O}(1)|$ be a line, $A$ is a globally generated line bundle on $C$ and $V$ be as earlier. Again, the dual LM bundle $F$ corresponding to $(C, A, V)$ is $\mu_{\mathcal{O}(1)}$-stable. By Corollary 4.3, the dual parabolic LM bundle $\left(F, \mathfrak{F}_{C}, a_{1}, a_{2}\right)$ is parabolic $\mu_{\mathcal{O}(1)}$-stable for any weights $a_{i}$ such that $a_{2}-a_{1}<1$. Set $a_{1}=0$ and $a_{2}=\frac{N-1}{N}$, i.e. $m=1$ in part (a).

By part (a) the dual LM bundle $F^{\prime}$ on $Y$ corresponding to the triple $\left(C^{\prime}, A^{\prime}, V\right)$ is $\mu_{p^{*} \mathcal{O}(1)}$-semistable on $Y$. Let $l=\operatorname{deg} A^{\prime}$. There is a flat family of LM bundles on $Y$ parametrized by an open subset of the elements of the Brill-Noether variety $\mathcal{G}_{l}^{1}\left(\left|\mathcal{O}_{Y}\left(C^{\prime}\right)\right|\right)$, cf. [18, $\left.\S 3\right]$. Since semistability of vector bundles is an open condition in flat families [8, Prop. 2.3.1], there is an irreducible component of $\mathcal{G}_{l}^{1}\left(\left|\mathcal{O}_{Y}\left(C^{\prime}\right)\right|\right)$ corresponding to $\mu_{p^{*} \mathcal{O}(1)^{-}}$semistable LM bundles.

(b') We consider a particular case of (b), where $L$ additionally satisfies $L^{2}=2$. Then a general curve $C \in|L|$ has genus 2, Clifford dimension 1 and maximal gonality 
$k$. Suppose $A$ is a complete base-point free $g_{d}^{1}$ on $C$ where $d$ is a positive integer such that the Brill-Noether number $\rho(2,1, d)>0$ (note that $d=3$ satisfies the requirement that $\rho(2,1, d)>0$ and completeness). Then the dual LM bundle $F:=F_{C, A}$ associated to a general $C$ and $A$ is $\mu_{L}$-stable. By Corollary 4.2 , the dual parabolic bundle $\left(F, \mathfrak{F}_{C}, a_{1}, a_{2}\right)$ is parabolic $\mu_{L}$-stable for any weights $a_{i}$ such that $a_{2}-a_{1}<\frac{1}{g-1}=1$.

Set weights $a_{1}=0$ and $a_{2}=\frac{N-1}{N}$, i.e. $m=1$ in case (b). Then $\left(F, \mathfrak{F}_{C}, 0, \frac{N-1}{N}\right)$ is

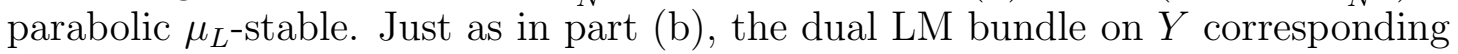
to the triple $\left(C^{\prime}, A^{\prime}, H^{0}(C, A)\right)$ is $\mu_{p^{*} L^{-}}$-semistable. Consider the flat family of LM bundles parametrized by the elements of an open subset of the Brill-Noether variety $\mathcal{G}_{l}^{1}\left(\left|\mathcal{O}_{Y}\left(C^{\prime}\right)\right|\right)$, cf. [18, $\left.\S 3\right]$. Here $l=\operatorname{deg} A^{\prime}$. Since semistability of vector bundles is an open condition in flat families [8, Prop. 2.3.1], there is an irreducible

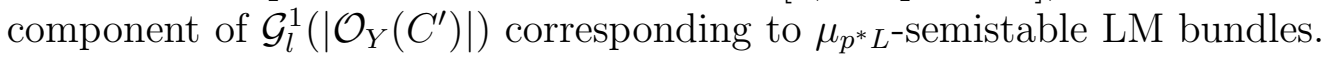

Remark 6.2. Consider $\sigma: X \rightarrow \mathbb{P}^{2}$, a K3 surface which is a double cover of the projective plane branched along a smooth sextic curve, and $L=\sigma^{*} \mathcal{O}_{\mathbb{P}^{2}}(1)$. Then $X$ and $L$ satisfy the requirements of part ( $\left.b^{\prime}\right)$ of the above theorem. That is, $\left(L^{2}\right)=2$ and a general curve $C \in|L|$ has genus 2, Clifford dimension 1 and constant gonality 2, cf. [5, Theorem A and Prop. 3.3].

\section{REFERENCES}

[1] M. Aprodu and G. Farkas, Green's conjecture for curves on arbitrary K3 surfaces, Compos. Math. $\mathbf{1 4 7}(3), 839-851,2011$.

[2] U. Bhosle, Parabolic sheaves on higher dimensional varieties, Math. Ann. 293, 177-192, 1992.

[3] I. Biswas, Parabolic bundles as orbifold bundles, Duke Math. J., 88(2), 305-325, 1997.

[4] R. Brussee, Stable bundles on blown up surfaces, Math. Z., 205, 551-565, 1990.

[5] C. Ciliberto and G. Pareschi, Pencils of minimal degree on curves on a K3 surface, J. Reine Angew. Math., 460, 15-36, 1995.

[6] M. Green and R. Lazarsfeld, Special divisors on curves on a K3 surface, Invent. Math., 89(2), 357-370, 1987.

[7] R. Hartshorne, Stable Reflexive Sheaves, Math. Ann. 254 (2), 121-176, 1980.

[8] D. Huybrechts and M. Lehn, The geometry of moduli spaces of sheaves, 2nd ed., Cambridge University Press, Cambridge, 2010.

[9] A. L. Knutsen, On two conjectures for curves on K3 surfaces, Int. J. Math. 20(12), 1547-1560, 2009.

[10] A. Krug, Extension groups of tautological sheaves on Hilbert schemes, J. Algebraic Geom., 23(3), 571-598, 2014.

[11] R. Lazarsfeld, Brill-Noether-Petri without degenerations, J. Diff. Geom. 23, 299-307, 1986.

[12] R. Lazarsfeld, Positivity in algebraic geometry I $\&$ II, Ergebnisse der Mathematik und ihrer Grenzgebiete, vol. 48 \& 49, Springer-Verlag, Berlin, 2004.

[13] M. Lelli-Chiesa, Stability of rank-3 Lazarsfeld-Mukai bundles on K3 surfaces, Proc. Lond. Math. Soc. 107 (3), 451-479, 2013.

[14] M. Maruyama and K. Yokogawa, Moduli of parabolic stable sheaves, Math. Ann., 293(1), 77-99, 1992.

[15] V. B. Mehta and C. S. Seshadri, Moduli of vector bundles on curves with parabolic structures, Math. Ann., 248(3), 205-239, 1980.

[16] S. Mukai, Biregular classification of Fano threefolds and Fano manifolds of coindex 3, Proc. Nat. Acad. Sci. USA, 86, 3000-3002, 1989.

[17] T. Nakashima, Moduli of stable bundles on blown up surfaces, J. Math. Kyoto Univ., 571-581, 1993.

[18] P. Narayanan, On the semistability of certain Lazarsfeld-Mukai bundles on abelian surfaces, Annali dell'Universita di Ferrara, 2017, doi:10.1007/s11565-017-0277-z. 
[19] P. Narayanan, Lazarsfeld-Mukai reflexive sheaves and their stability, Communications in Algebra, 2017, doi:10.1080/00927872.2017.1354009.

[20] C. S. Seshadri, Moduli of vector bundles on curves with parabolic structures, Bull. Amer. Math. Soc., 83(1), 124-126, 1977.

[21] C. Voisin, Greens generic syzygy conjecture for curves of even genus lying on a K3 surface, J. European Math. Soc. 4, 363-404, 2002.

[22] C. Voisin, Greens canonical syzygy conjecture for generic curves of odd genus, Compositio Math. 141, 1163-1190, 2005.

Department of Mathematics, Indian Institute of Technology Madras, Chennai - 600036.

E-mail address: poorna.p.narayanan@gmail.com 